\title{
Vector Valued Polynomials, Exponential Polynomials and Vector Valued Harmonic Analysis
}

\author{
M. Laczkovich
}

\begin{abstract}
Let $G$ be a topological Abelian semigroup with unit, and let $E$ be a Banach space. We define, for functions mapping $G$ into $E$, the classes of polynomials, generalized polynomials, local polynomials, exponential polynomials, and some other relevant classes. We establish their connections with each other and find their representations in terms of the corresponding complex valued classes. We also investigate spectral synthesis and analysis in the class $C(G, E)$ of continuous functions $f: G \rightarrow E$. It is known that if $G$ is a compact Abelian group and $E$ is a Banach space, then spectral synthesis holds in $C(G, E)$. We give a self-contained proof of this fact, independent of the theory of almost periodic functions. On the other hand, we show that if $G$ is an infinite and discrete Abelian group and $E$ is a Banach space of infinite dimension, then even spectral analysis fails in $C(G, E)$. We also prove that if $G$ is discrete, has finite torsion free rank and if $E$ is a Banach space of finite dimension, then spectral synthesis holds in $C(G, E)$.
\end{abstract}

Mathematics Subject Classification. Primary 39B52; Secondary 22A20.

Keywords. Banach space valued polynomials, exponential polynomials, spectral synthesis.

\section{Introduction}

Let $G$ be a topological Abelian semigroup with unit. For complex valued functions defined on $G$, the classes of polynomials, generalized polynomials, local

Supported by the Hungarian National Foundation for Scientific Research, Grant No. K124749. 
polynomials, exponential polynomials have been defined and their basic properties have been established. (See, e.g., [5-8,10-12,14-16] and the references therein.) Our first aim is to extend these notions to the vector valued case.

Let $E$ be a Banach space, and let $C(G, E)$ denote the set of continuous functions $f: G \rightarrow E$. A function $f \in C(G, E)$ is a generalized polynomial, if there is an $n \geq 0$ such that $\Delta_{h_{1}} \ldots \Delta_{h_{n+1}} f=0$ for every $h_{1}, \ldots, h_{n+1} \in G$, where $\Delta_{h}$ is the difference operator. We say that $f \in C(G, E)$ is a polynomial, if it is a generalized polynomial, and the linear span of its translates is of finite dimension; $f$ is a w-polynomial, if $u \circ f$ is a polynomial for every $u \in E^{*}$, and $f$ is a local polynomial, if it is a polynomial on every finitely generated subsemigroup. We show that each of the classes of polynomials, w-polynomials, generalized polynomials, local polynomials is contained in the next class (Theorem 8). We also prove that if $G$ is an Abelian group and has a dense subgroup with finite torsion free rank, then these classes coincide (see Theorem 9).

We introduce the classes of exponential polynomials and w-exponential polynomials as well, establish their representations and connection with polynomials and w-polynomials.

We also investigate spectral synthesis and analysis in the class $C(G, E)$. It is known that if $G$ is a compact Abelian group and $E$ is a Banach space, then spectral synthesis holds in $C(G, E)$. In the appendix we give a self-contained proof of this fact, independent of the theory of almost periodic functions. As we show, the situation for locally compact Abelian groups is different. We prove that if $G$ is an infinite and discrete Abelian group and $E$ is a Banach space of infinite dimension, then even spectral analysis fails in $C(G, E)$ (see Theorem 17). If, however, $G$ is discrete, has finite torsion free rank and if $E$ is a Banach space of finite dimension, then spectral synthesis holds in $C(G, E)$ (see Theorem 18).

\section{Vector Valued Polynomials and Exponential Polynomials}

Let $G$ be a topological Abelian semigroup with unit. We denote the semigroup operation by addition, and denote the unit by 0 . Let $E$ be a Banach space over the complex field $\mathbb{C}$. We denote by $E^{G}$ the set of maps from $G$ into $E$, and by $C(G, E)$ the set of continuous functions $f: G \rightarrow E$.

Let $T_{g}$ denote the translation operator on $E^{G}$ defined by $T_{g} f(x)=f(x+$ $g$ ) for every $f \in E^{G}$ and $g, x \in G$. A subset $V \subset E^{G}$ is translation invariant, if $T_{g} f \in V$ whenever $f \in V$ and $g \in G$. If $f \in E^{G}$, then $L_{f}$ denotes the linear span of $\left\{T_{g} f: g \in G\right\}$.

The operator $\Delta_{g}$ is defined by $\Delta_{g}=T_{g}-T_{0}$. That is, we have $\Delta_{g} f(x)=$ $f(x+g)-f(x)$ for every $f \in E^{G}$ and $x \in G$.

We say that a continuous function $f \in C(G, E)$ is a generalized polynomial $^{1}$, if there is an $n \geq 0$ such that $\Delta_{h_{1}} \ldots \Delta_{h_{n+1}} f=0$ for every

\footnotetext{
${ }^{1}$ Our terminology differs from that of [15].
} 
$h_{1}, \ldots, h_{n+1} \in G$. The smallest $n$ with this property is the degree of $f$, denoted by $\operatorname{deg} f$. The degree of the identically zero function is -1 .

By Djoković's theorem [5] (see also [6, Section 6]), a continuous function $f \in C(G, E)$ is a generalized polynomial if and only if $f=\sum_{i=1}^{n} f_{i}$, where $f_{i}$ is a monomial of degree $i$ for every $i=1, \ldots, n$, and $f_{0}$ is constant. By a monomial of degree $i$ we mean a function of the form $A(x, \ldots, x)$, where $A\left(x_{1}, \ldots, x_{i}\right)$ is a map from $G^{i}$ to $E$ which is symmetric, and $i$-additive; that is, additive in each variable. It is easy to see that the representation $f=\sum_{i=1}^{n} f_{i}$ is unique.

It is clear that the set of generalized polynomials forms a linear subspace of $C(G, E)$ over $\mathbb{C}$.

Theorem 1. (i) A continuous function $f \in C(G, E)$ is a generalized polynomial if and only if $u \circ f$ is a (complex valued) generalized polynomial for every $u \in E^{*}$.

(ii) If $f$ is a generalized polynomial, then $\operatorname{deg}(u \circ f) \leq \operatorname{deg} f$ for every $u \in E^{*}$.

(iii) If $f$ is a generalized polynomial, then there is an $u \in E^{*}$ such that $\operatorname{deg}(u \circ$ $f)=\operatorname{deg} f$.

Proof. The "only if" direction of (i) is obvious, and so is (ii). To prove the "if" statement of (i), let $E_{n}^{*}$ denote the set of linear functionals $u \in E^{*}$ such that $\Delta_{h_{1}} \ldots \Delta_{h_{n+1}}(u \circ f)=0$ for every $h_{1}, \ldots, h_{n+1} \in G$. It is easy to see that $E_{n}^{*}$ is a closed linear subspace of $E^{*}$ for every $n=0,1, \ldots$.

If $u \circ f$ is a generalized polynomial for every $u \in E^{*}$, then $E^{*}=\bigcup_{n=0}^{\infty} E_{n}^{*}$. Then, by the Baire category theorem, there is an $n$ such that int $E_{n}^{*} \neq \emptyset$, and thus $E_{n}^{*}=E^{*}$. Let $n$ be the smallest such $n$.

We show that $\Delta_{h_{1}} \ldots \Delta_{h_{n+1}} f=0$ for every $h_{1}, \ldots, h_{n+1}$. Indeed, if $\Delta_{h_{1}} \ldots \Delta_{h_{n+1}} f(x) \neq 0$ for some $h_{1}, \ldots, h_{n+1}, x \in G$, then there is an $u \in E^{*}$ such that

$$
\Delta_{h_{1}} \ldots \Delta_{h_{n+1}}(u \circ f)(x)=u\left(\Delta_{h_{1}} \ldots \Delta_{h_{n+1}} f(x)\right) \neq 0,
$$

which is impossible. This proves both (i) and (iii).

Remark 2. The continuity of the function $f$ cannot be omitted from the conditions of the theorem. In other words, a function $f \in E^{G}$ such that $u \circ f$ is a complex valued generalized polynomial, hence continuous for every $u \in E^{*}$ is not necessarily continuous itself.

As an example, let $E$ be an infinite dimensional Banach space, and let $G$ denote the vector space of $E$ endowed with the weak topology of $E$. Then $G$ is a topological vector space. Let $f$ denote the identity on $G$ as a map from $G$ to the Banach space $E$. Then $f$ is not continuous, as the original topology of $E$ is strictly stronger than the weak topology. On the other hand, if $u \in E^{*}$, then $u \circ f=u$ is a continuous additive function, therefore, a generalized polynomial.

We show that if $G$ is a normed linear space, then the continuity of $f$ is a consequence of the other condition. 
Theorem 3. Let $G$ be a normed linear space, and let $E$ be a Banach space. $A$ function $f \in E^{G}$ is a generalized polynomial if and only if $u \circ f$ is a (complex valued) generalized polynomial for every $u \in E^{*}$.

Proof. By Theorem 1, $f$ is a generalized polynomial w.r.t. the discrete topology. Thus we only have to show that $f$ is continuous.

Let $f=\sum_{i=0}^{n} f_{i}$, where $f_{i}$ is a monomial of degree $i$ for $1 \leq i \leq n$, and $f_{0}$ is a constant. It is enough to show that $f_{i}$ is continuous for every $i=1, \ldots, n$.

It is easy to see that $f(k x)=\sum_{i=0}^{n} k^{i} \cdot f_{i}(x)$ for every $x \in G$ and for every positive integer $k$. These equations for $k=1, \ldots, n+1$ constitute a linear system of equations with unknowns $f_{i}(x)(i=0, \ldots, n)$. Since the determinant of this system is nonzero (being a Vandermonde determinant), it follows that each $f_{i}(x)$ is a linear combination of $f(x), \ldots, f((n+1) x)$ with rational coefficients.

If $u \in E^{*}$, then $u \circ f$ is a generalized polynomial, hence continuous. Then each of the functions $x \mapsto u(f(k x))(k=1, \ldots, n+1)$ is continuous, and thus $u \circ f_{i}$, being a linear combinations of these functions, is also continuous for every $i=1, \ldots, n$.

Let $B_{r}$ denote the open ball $\left\{x \in G:\|x\|_{G}<r\right\}$ (recall that $G$ is a normed linear space by assumption). Let $1 \leq i \leq n$ be fixed. We show that $f_{i}\left(B_{1}\right)$ is weakly bounded in $E$. Indeed, if $u \in E^{*}$, then the continuity of $u \circ f_{i}$ implies that for a suitable positive integer $k,\left|u\left(f_{i}(x)\right)\right|<1$ for every $x \in B_{1 / k}$. Therefore, if $x \in B_{1}$, then $x / k \in B_{1 / k},\left|u\left(f_{i}(x / k)\right)\right|<1$ and $\left|u\left(f_{i}(x)\right)\right|<k^{i}$, showing that $u$ is bounded on $f_{i}\left(B_{1}\right)$. This proves that $f_{i}\left(B_{1}\right)$ is weakly bounded in $E$. Since, in a Banach space, every weakly bounded set is originally bounded [13, 3.18 Theorem], it follows that $\|y\|_{E}<K$ for every $y \in f_{i}\left(B_{1}\right)$ with a suitable positive integer $K$.

If $\varepsilon>0$ is given, then there is an integer $m$ such that $m>K / \varepsilon$. If $x \in B_{1 / m}$, then

$$
\left\|f_{i}(x)\right\|_{E}=\left\|m^{-i} f_{i}(m x)\right\|_{E}<K / m^{i}<\varepsilon,
$$

proving that $f_{i}$ is continuous at zero. Now, it is known that if a monomial is continuous at one point, then it is continuous everywhere. See [15, Theorem 3.6]. Note that monomials are "algebraic polynomials" in the terminology of [15], and that the conditions of [15, Theorem 3.6] are satisfied if $G$ is a normed linear space and $E$ is a Banach space. Thus $f_{i}$ is continuous on $G$ for every $i=1, \ldots, n$, and this is what we wanted to show.

A function $f \in \mathbb{C}^{G}$ is said to be a polynomial, if there are continuous additive functions $a_{1}, \ldots, a_{n}: G \rightarrow \mathbb{C}$ and there is a $P \in \mathbb{C}\left[x_{1}, \ldots, x_{n}\right]$ such that $f=P\left(a_{1}, \ldots, a_{n}\right)$. It is well-known that every complex valued polynomial is a generalized polynomial.

A continuous function $m: G \rightarrow \mathbb{C}$ is called an exponential function, if $m \neq 0$ and $m(x+y)=m(x) \cdot m(y)$ for every $x, y \in G$.

A function $f \in \mathbb{C}^{G}$ is an exponential polynomial, if there are polynomials $p_{1}, \ldots, p_{n}: G \rightarrow \mathbb{C}$ and exponentials $m_{1}, \ldots, m_{n}$ such that $f=\sum_{i=1}^{n} p_{i} \cdot m_{i}$. 
It is well-known that a continuous function $f \in C(G, \mathbb{C})$ is an exponential polynomial if and only if $\operatorname{dim} L_{f}<\infty$. (For Abelian semigroups see [12], for Abelian groups see [14] and [15, Theorem 10.2]. See also [9] for the history of the theorem and for a simple proof.) In possession of this result the following definition seems reasonable. We say that a continuous function $f \in C(G, E)$ is an exponential polynomiat ${ }^{2}$, if $\operatorname{dim} L_{f}<\infty$.

Theorem 4. A function $f \in C(G, E)$ is an exponential polynomial if and only if there are finitely many complex valued exponential polynomials $f_{1}, \ldots, f_{k} \in$ $C(G, \mathbb{C})$ and elements $e_{1}, \ldots, e_{k} \in E$ such that $f=f_{1} \cdot e_{1}+\ldots+f_{k} \cdot e_{k}$.

Proof. The "if" statement is clear: if $f_{1}, \ldots, f_{k}$ are exponential polynomials and $f=f_{1} \cdot e_{1}+\ldots+f_{k} \cdot e_{k}$, then $L_{f_{1} \cdot e_{1}}, \ldots, L_{f_{k} \cdot e_{k}}$ are of finite dimension, and then so is $L_{f}$.

To prove the converse, suppose that $\operatorname{dim} L_{f}<\infty$. First we show that the linear subspace $F$ of $E$ generated by $R(f)$, the range of $f$, is of finite dimension. Suppose not. Then there are elements $x_{1}, \ldots, x_{n} \in G$ such that $n>\operatorname{dim} L_{f}$, and $f\left(x_{1}\right), \ldots, f\left(x_{n}\right)$ are linearly independent over $\mathbb{C}$. Now $n>\operatorname{dim} L_{f}$ implies that $T_{x_{1}} f, \ldots, T_{x_{n}} f$ are linearly dependent, and thus $\sum_{i=1}^{n} c_{i} T_{x_{i}} f=0$ for some complex numbers $c_{1}, \ldots, c_{n}$, not all zero. Then $\sum_{i=1}^{n} c_{i} f\left(x+x_{i}\right)=0$ for every $x$. In particular, putting $x=0$ we get $\sum_{i=1}^{n} c_{i} f\left(x_{i}\right)=0$, which contradicts the fact that $f\left(x_{1}\right), \ldots, f\left(x_{n}\right)$ are linearly independent.

This proves that $\operatorname{dim} F<\infty$. Let $e_{1}, \ldots, e_{k}$ be a basis of $F$. Then there are functions $f_{1}, \ldots, f_{k}: G \rightarrow \mathbb{C}$ such that $f=f_{1} \cdot e_{1}+\ldots+f_{k} \cdot e_{k}$ on $G$. Since $e_{1}, \ldots, e_{k}$ are linearly independent, there are linear functionals $u_{1}, \ldots, u_{k} \in E^{*}$ such that $u_{i}\left(e_{i}\right)=1$ and $u_{i}\left(e_{j}\right)=0$ for every $1 \leq i, j \leq k, i \neq j$. Thus $f_{i}=u_{i} \circ f$ for every $i=1, \ldots, k$. Since $f$ is continuous, we can see that so are $f_{1}, \ldots, f_{k}$. Also, we have $\operatorname{dim} L_{f_{i}}=\operatorname{dim} L_{u_{i} \circ f} \leq \operatorname{dim} L_{f}<\infty$, and thus $f_{i}$ is an exponential polynomial for every $i=1, \ldots, k$.

The definition of polynomials cannot be generalized to the vector-valued case, as Banach spaces are not algebras. However, the following observation makes it clear what a reasonable generalization could be.

Proposition 5. A complex valued function $f: G \rightarrow \mathbb{C}$ is a polynomial if and only if $f$ is a generalized polynomial, and $\operatorname{dim} L_{f}<\infty$.

Proof. We noted already that every polynomial is a generalized polynomial. In fact, one can prove by induction on $\operatorname{deg} P$ that if $f=P\left(a_{1}, \ldots, a_{n}\right)$, where $P \in \mathbb{C}\left[x_{1}, \ldots, x_{n}\right]$ and $a_{1}, \ldots, a_{n}: G \rightarrow \mathbb{C}$ are continuous additive functions, then $f$ is a generalized polynomial of degree at most $\operatorname{deg} P$. Similarly, $\operatorname{dim} L_{f}<$ $\infty$ can also be proved by induction on $\operatorname{deg} P$. Or, we can argue that if $f$ is a polynomial, then it is an exponential polynomial, since $f=f \cdot 1$ and the

\footnotetext{
${ }^{2}$ Our terminology differs from that of [3, Definition 1.3, p. 366]. As we can see in Theorem 4, a function is an exponential polynomial in our sense if and only if it is a finite sum of exponential polynomials in the sense of [3].
} 
identically 1 function is an exponential. Thus $\operatorname{dim} L_{f}<\infty$ follows from a theorem quoted before. This proves the "only if" part of the proposition.

Now suppose that $f$ is a generalized polynomial and $\operatorname{dim} L_{f}<\infty$. The latter condition implies that $f$ is an exponential polynomial; that is, $f=$ $\sum_{i=1}^{k} p_{i} \cdot m_{i}$, where $p_{1}, \ldots, p_{k}$ are polynomials and $m_{1}, \ldots, m_{k}$ are exponentials. We may assume that $p_{1}, \ldots, p_{k}$ are nonzero and $m_{1}, \ldots, m_{k}$ are distinct.

It is known that the representation of a function $f: G \rightarrow \mathbb{C}$ in the form $\sum_{i=1}^{s} p_{i} \cdot m_{i}$, where $m_{1}, \ldots, m_{s}$ are distinct exponentials and $p_{1}, \ldots, p_{s}$ are nonzero generalized polynomials is unique (if exists). For Abelian groups this is proved in [15, Lemma 4.3, p. 41] and in [7, Lemma 6]. It is easy to check that the proof of [7, Lemma 6] works in Abelian semigroups as well. The uniqueness follows also from Lemma 15 below.

In our case $f \cdot 1=\sum_{i=1}^{s} p_{i} \cdot m_{i}$ and thus the uniqueness of the representation implies $s=1, m_{1}=1$ and $f=p_{1}$. Thus $f$ is a polynomial, which proves the "if" part of the proposition.

The proposition above motivates the following definition: a function $f \in$ $C(G, E)$ is a polynomial, if $f$ is a generalized polynomial, and $\operatorname{dim} L_{f}<\infty$.

Theorem 6. A function $f \in E^{G}$ is a polynomial if and only if there are finitely many complex valued polynomials $f_{1}, \ldots, f_{k}$ and elements $e_{1}, \ldots, e_{k} \in E$ such that $f=f_{1} \cdot e_{1}+\ldots+f_{k} \cdot e_{k}$.

Proof. Suppose that $f=f_{1} \cdot e_{1}+\ldots+f_{k} \cdot e_{k}$, where $f_{1}, \ldots, f_{k}$ are complex valued polynomials and $e_{1}, \ldots, e_{k} \in E$. Then $f_{1}, \ldots, f_{k}$ are continuous, hence so is $f$. Also, $f_{1}, \ldots, f_{k}$ are generalized polynomials, hence so are $f_{1} \cdot e_{1}, \ldots, f_{k} \cdot e_{k}$, and then so is $f$. Also, $L_{f_{1}}, \ldots, L_{f_{k}}$ are of finite dimension, implying $\operatorname{dim} L_{f}<\infty$. This proves the "if" statement.

If $f$ is a polynomial, then $\operatorname{dim} L_{f}<\infty$. By Theorem 4, this implies that $f=f_{1} \cdot e_{1}+\ldots+f_{k} \cdot e_{k}$, where $f_{1}, \ldots, f_{k}$ are complex valued exponential polynomials, and $e_{1}, \ldots, e_{k} \in E$. We may assume that $e_{1}, \ldots, e_{k}$ are linearly independent. Then there are linear functionals $u_{1}, \ldots, u_{k} \in E^{*}$ such that $u_{i}\left(e_{i}\right)=1$ and $u_{i}\left(e_{j}\right)=0$ for every $1 \leq i, j \leq k, i \neq j$. Thus $f_{i}=u_{i} \circ f$ for every $i=1, \ldots, k$. Since $f$ is a generalized polynomial, we can see that so are $f_{1}, \ldots, f_{k}$. Summing up: $f_{1}, \ldots, f_{k}$ are generalized polynomials and exponential polynomials. Therefore, they are polynomials by Proposition 5 .

Theorem 7. A function $f \in E^{G}$ is an exponential polynomial if and only if $f=\sum_{i=1}^{k} m_{i} \cdot p_{i}$, where $m_{1}, \ldots, m_{k}$ are (complex valued) exponentials, and $p_{1}, \ldots, p_{k} \in E^{G}$ are polynomials.

Proof. If $m$ is an exponential and $p \in E^{G}$, then $T_{g}(m \cdot p)=m(g) \cdot m \cdot T_{g} p$, and thus

$$
T_{g}(m \cdot p) \in\left\{m \cdot \phi: \phi \in L_{p}\right\}
$$

for every $g \in G$. If $p$ is a polynomial, then $L_{p}$ is of finite dimension, and then so is $L_{m \cdot p}$. Thus $m \cdot p$ is an exponential polynomial whenever $m$ is a complex 
valued exponential and $p$ is a polynomial. From this observation the "if" part of the statement of the theorem is obvious.

To prove the "only if" part, let $f$ be an exponential polynomial. By Theorem $4, f=f_{1} \cdot e_{1}+\ldots+f_{k} \cdot e_{k}$, where $f_{1}, \ldots, f_{k}$ are complex valued exponential polynomials and $e_{1}, \ldots, e_{k} \in E$. Let $f_{i}=\sum_{j=1}^{n_{i}} p_{i j} \cdot m_{i j}$, where $p_{i j}$ is a complex valued polynomial and $m_{i j}$ is an exponential for every $j=$ $1, \ldots, n_{i}$. Then

$$
f=\sum_{i=1}^{k} \sum_{j=1}^{n_{i}} m_{i j} \cdot\left(p_{i j} \cdot e_{i}\right),
$$

where $p_{i j} \cdot e_{i} \in E^{G}$ is a polynomial for every $i, j$.

\section{The Classes of w-Polynomials and w-Exponential Polynomials}

In the complex valued case the continuous additive functions are automatically polynomials. In the vector valued setting this is not the case, as the following example shows.

Let $E$ be an infinite dimensional Banach space, and let $G$ be its additive group with the same topology. Let $f: G \rightarrow E$ be the identity map on $G$. Then $f$ is a continuous additive function, but not a polynomial. Indeed, $L_{f}$ equals the set of functions $x \mapsto c x+e$, where $c \in \mathbb{C}$ and $e \in E$. In particular, $L_{f}$ contains the constant functions, and thus $\operatorname{dim} L_{f}=\infty$. Consequently, $f$ is not a polynomial. On the other hand, it is clear that $u \circ f=u$ is a (complex valued) polynomial for every $u \in E^{*}$. This motivates the following definition.

Let $G$ be a topological Abelian semigroup with unit, and let $E$ be a Banach space over the complex field $\mathbb{C}$. We say that a continuous function $f \in C(G, E)$ is a w-polynomial, if $u \circ f$ is a (complex valued) polynomial for every $u \in E^{*}$.

We introduce one more variation on the theme of polynomials. A continuous function $f \in C(G, E)$ is called a local polynomial, if the restriction of $f$ to any finitely generated subsemigroup of $G$ is a polynomial.

Theorem 8. Consider the following properties that a continuous function $f \in$ $C(G, E)$ may have:

(i) $f$ is a polynomial,

(ii) $f$ is a w-polynomial,

(iii) $f$ is a generalized polynomial,

(iv) $f$ is a local polynomial.

Then we have (i) $\Longrightarrow($ ii $) \Longrightarrow($ iii $) \Longrightarrow($ iv) .

Proof. (i) $\Longrightarrow$ (ii): If $f$ is a polynomial, then $f$ is a generalized polynomial, and $\operatorname{dim} L_{f}<\infty$. It is clear that if $u \in E^{*}$, then $u \circ f$ has the same properties, and thus $u \circ f$ is a polynomial by Proposition 5 . 
(ii) $\Longrightarrow$ (iii): If $f$ is a w-polynomial, then $u \circ f$ is a polynomial for every $u \in$ $E^{*}$. Thus $u \circ f$ is a generalized polynomial for every $u \in E^{*}$. Therefore, by Theorem $1, f$ is a generalized polynomial.

(iii) $\Longrightarrow($ iv): Let $f$ be a generalized polynomial. As we mentioned earlier, this implies, by Djoković's theorem [5], that $f=\sum_{i=1}^{n} f_{i}$, where $f_{i}$ is a monomial of degree $i$ for every $i=1, \ldots, n$, and $f_{0}$ is constant. Let $f_{i}(x)=A_{i}(x, \ldots, x)$, where $A_{i}\left(x_{1}, \ldots, x_{i}\right)$ is symmetric and additive in each variable.

Let $H$ be a finitely generated subsemigroup of $G$, and let $h_{1}, \ldots, h_{k}$ be a generating system of $H$. It is clear that the restriction $\left.f\right|_{H}$ is also a generalized polynomial. We prove that $\operatorname{dim} L_{f \mid H}<\infty$.

If $x, y \in H$, then $f(x+y)=\sum_{i=1}^{n} f_{i}(x+y)$. Since $A_{i}$ is symmetric and additive in each variable, we have $f_{i}(x+y)=A_{i}(x+y, \ldots, x+y)=$ $\sum_{j=0}^{i} g_{i}(x, y)$, where

$$
g_{i}(x, y)=\left(\begin{array}{l}
i \\
j
\end{array}\right) A(\underbrace{x, \ldots, x}_{j} \underbrace{y, \ldots, y}_{i-j}) .
$$

Since $y$ is a linear combinations with nonnegative integer coefficients of the elements $h_{1}, \ldots, h_{k}$, it follows that $g_{i}(x, y)$ is a linear combinations with nonnegative integer coefficients of the functions $A_{i}\left(x, \ldots, x, h_{\nu_{1}}, \ldots, h_{\nu_{j-i}}\right)$, where $\nu_{1}, \ldots, \nu_{j-i} \in\{1, \ldots, k\}$. Thus $T_{y} f_{i}$ is the linear combination of finitely many functions that are independent of the choice of $y \in H$. Therefore, $\operatorname{dim} L_{f_{i} \mid H}<\infty$ for every $i=1, \ldots, n$, and thus $\operatorname{dim} L_{f \mid H}<\infty$. This proves that $f$ is a polynomial on $H$. Since $H$ was an arbitrary finitely generated subsemigroup of $G$, it follows that $f$ is a local polynomial on $G$.

Note that if $E$ is finite dimensional, then every $w$-polynomial is a polynomial. Indeed, if $f$ is a $w$-polynomial and $e_{1}, \ldots, e_{n}$ is a basis of $E$, then $f=f_{1} \cdot e_{1}+\ldots+f_{n} \cdot e_{n}$, where $f_{1}, \ldots, f_{n} \in \mathbb{C}^{G}$. The argument of the proof of Theorem 6 gives that $f_{i}=u_{i} \circ f$ with suitable $u_{1}, \ldots, u_{n} \in E^{*}$, and thus $f_{1}, \ldots, f_{n}$ are polynomials. Thus $f$ itself is a polynomial by Theorem 6 .

If $G$ is a finitely generated semigroup, then every local polynomial is a polynomial, and thus properties (i)-(iv) are equivalent. We show that for Abelian groups somewhat more is true. If $G$ is an Abelian group, then we denote by $r_{0}(G)$ the torsion free rank of $G$; that is, the cardinality of a maximal independent system of elements of infinite order.

Theorem 9. Let $G$ be a topological Abelian group, and suppose that there is a dense subgroup $H$ of $G$ such that $r_{0}(H)<\infty$. Then properties (i)-(iv) listed in Theorem 8 are equivalent.

Proof. First we assume that $r_{0}(G)<\infty$. Then there is a finitely generated subgroup $H$ of $G$ such that the factor group $G / H$ is torsion. In other words, for every $h \in G$ there is a positive integer $k$ such that $k h \in H$.

It is enough to prove that if $f \in C(G, E)$ is a local polynomial, then $f$ is a polynomial. 
First we show that $f$ is a generalized polynomial. Since $f$ is a local polynomial, the restriction $\left.f\right|_{H}$ to the finitely generated subgroup $H$ is a polynomial. In particular, $\left.f\right|_{H}$ is a generalized polynomial, and thus $\left.f\right|_{H}=\sum_{i=1}^{n} f_{i}$, where $f_{i}$ is a monomial of degree $i$ for every $i=1, \ldots, n$, and $f_{0}$ is constant.

We show that $f$ is a generalized polynomial of degree $n$ on $G$. Let $a_{1}, \ldots, a_{n+1}, x \in G$ be arbitrary; we prove $\Delta_{a_{1}} \ldots \Delta_{a_{n+1}} f(x)=0$. Let $\bar{H}$ denote the subgroup of $G$ generated by $H$ and the elements $a_{1}, \ldots, a_{n+1}, x$. Then $\bar{H}$ is finitely generated. Since $f$ is a local polynomial, it follows that $\left.f\right|_{\bar{H}}$ is a polynomial, hence a generalized polynomial. Thus $\left.f\right|_{\bar{H}}=\sum_{j=1}^{m} g_{j}$, where $g_{i}$ is a monomial of degree $j$ for every $j=1, \ldots, m$, and $g_{0}$ is constant. We may assume that $g_{m}$ is not identically zero on $G$. We prove $m \leq n$.

Since $H \subset \bar{H}$, we have $\left.f\right|_{H}=\left.\sum_{j=1}^{m} g_{j}\right|_{H}$. Now the representation of $\left.f\right|_{H}$ as a sum of monomials is unique. If $m>n$, then necessarily $\left.g_{m}\right|_{H}=0$. Let $g_{m}(x)=B(x, \ldots, x)$, where $B$ is $m$-additive. If $h \in \bar{H}$, then $k h \in H$ with a suitable positive integer $k$. Then

$$
g_{m}(h)=B(h, \ldots, h)=k^{-m} B(k h, \ldots, k h)=k^{-m} g_{m}(k h)=0,
$$

as $k h \in H$. Thus $g_{m}$ is identically zero on $\bar{H}$, which is a contradiction.

This proves $m \leq n$. Then $\left.f\right|_{\bar{H}}=\sum_{j=1}^{m} g_{j}$ implies that $\left.f\right|_{\bar{H}}$ is a generalized polynomial of degree at most $n$. Since $a_{1}, \ldots, a_{n+1}, x \in \bar{H}$, we obtain $\Delta_{a_{1}} \ldots \Delta_{a_{n+1}} f(x)=0$, and this is what we wanted to show. Thus $f$ is a generalized polynomial on $G$. Let $\left.f\right|_{H}=\sum_{i=1}^{n} f_{i}$, where $f_{i}$ is a monomial of degree $i$ for every $i=1, \ldots, n$, and $f_{0}$ is constant.

Now we prove that $f$ is a polynomial. We only have to show that $\operatorname{dim} L_{f}<$ $\infty$.

Clearly, it is enough to show that $\operatorname{dim} L_{f_{i}}<\infty$ for every $i=1, \ldots, n$. Let $f_{i}(x)=A_{i}(x, \ldots, x)$, where $A_{i}$ is symmetric and $i$-additive. Let $h_{1}, \ldots, h_{N}$ be a generating system of $H$. We prove that $L_{f_{i}}$ is contained by the linear hull of the functions

$$
A(\underbrace{x, \ldots, x}_{i-j}, h_{\nu_{1}}, \ldots, h_{\nu_{j}})
$$

where $0 \leq j \leq i$ and $\nu_{1}, \ldots, \nu_{j} \in\{1, \ldots, N\}$. Since the number of these functions is finite, this will prove $\operatorname{dim} L_{f_{i}}<\infty$.

Let $h \in G$ be arbitrary. Then $T_{h} f_{i}(x)=f_{i}(x+h)=A_{i}(x+$ $h, \ldots, x+h)$ is a linear combination with integer coefficients of the functions $A(\underbrace{x, \ldots, x}_{i-j}, h, \ldots, h)(j=0, \ldots, i)$. Let $k$ be a positive integer with $k h \in H$. If $j$ is fixed, then

$$
A(\underbrace{x, \ldots, x}_{i-j}, h, \ldots, h)=k^{-j} A(\underbrace{x, \ldots, x}_{i-j}, k h, \ldots, k h) .
$$

Now $k h$, being an element of $H$, is a linear combination with integer coefficients of the elements $h_{1}, \ldots, h_{N}$. Since $A_{i}$ is additive in each variable, it follows from 
(2) that $A(\underbrace{x, \ldots, x}_{i-j}, h, \ldots, h)$ is a linear combination with rational coefficients of the function listed in (1). This completes the proof of $\operatorname{dim} L_{f_{i}}<\infty$. We proved that the statement of the theorem is true if $r_{0}(G)<\infty$.

Now we assume that $G$ has a dense subgroup $H$ such that $r_{0}(H)<\infty$. Suppose that $f \in C(G, E)$ is a local polynomial. We have to prove that $f$ is a polynomial; that is, a generalized polynomial satisfying $\operatorname{dim} L_{f}<\infty$.

Since $r_{0}(H)<\infty$ and $f$ is a local polynomial on $H$, it follows that $\left.f\right|_{H}$ is a polynomial on $H$. Then $\left.f\right|_{H}$ is a generalized polynomial on $H$; let $n=\left.\operatorname{deg} f\right|_{H}$. We show that $f$ is a generalized polynomial of degree $n$ on $G$. Let $a_{1}, \ldots, a_{n+1} \in G$ be arbitrary; we prove $\Delta_{a_{1}} \ldots \Delta_{a_{n+1}} f=0$. We have

$$
\Delta_{a_{1}} \ldots \Delta_{a_{n+1}} f(x)=\sum_{\vartheta}(-1)^{|\vartheta|+1} f\left(x+\vartheta_{1} a_{1}+\ldots+\vartheta_{n+1} a_{n+1}\right),
$$

where $\vartheta=\left(\vartheta_{1}, \ldots, \vartheta_{n+1}\right)$ runs through all $0-1$ sequences of length $n+1$, and $|\vartheta|=\sum_{i=1}^{n+1} \vartheta_{i}$. Let $x \in G$ and $\varepsilon>0$ be fixed. Since $f$ is continuous, there is a neighbourhood $U$ of zero such that

$$
\left\|f\left(x+\vartheta_{1} a_{1}+\ldots+\vartheta_{n+1} a_{n+1}\right)-f\left(x^{\prime}+\vartheta_{1} h_{1}+\ldots+\vartheta_{n+1} h_{n+1}\right)\right\|_{E}<\varepsilon
$$

for every $\vartheta$, whenever $x^{\prime} \in U+x$ and $h_{i} \in U+a_{i}(i=1, \ldots, n+1)$. Choosing elements $x^{\prime} \in(U+x) \cap H$ and $x_{i} \in\left(U+a_{i}\right) \cap H(i=1, \ldots, n+1)$, and noting that $\Delta_{x_{1}} \ldots \Delta_{x_{n+1}} f\left(x^{\prime}\right)=0$ by $\left.\operatorname{deg} f\right|_{H}=n$, we can see that $\left\|\Delta_{a_{1}} \ldots \Delta_{a_{n+1}} f(x)\right\|_{E}<2^{n+1} \varepsilon$. Since this is true for every $x \in G$ and $\varepsilon>0$, it follows that $\Delta_{a_{1}} \ldots \Delta_{a_{n+1}} f=0$. Thus $f$ is a generalized polynomial.

Since $\left.f\right|_{H}$ is a polynomial, $L_{f \mid H}$ is of finite dimension. Let $\left.T_{k_{1}} f\right|_{H}$ $, \ldots,\left.T_{k_{N}} f\right|_{H}$ be a basis of $L_{f \mid H}$, and let $V$ denote the linear hull of the functions $T_{k_{1}} f, \ldots, T_{k_{N}} f$. If $h \in H$, then $\left.T_{h} f\right|_{H} \in L_{f \mid H}$, and thus there are complex numbers $c_{1}, \ldots, c_{N}$ such that $\left.T_{h} f\right|_{H}=\left.\sum_{i=1}^{N} c_{i} T_{k_{i}} f\right|_{H}$; that is,

$$
f(x+h)=\sum_{i=1}^{N} c_{i} \cdot f\left(x+k_{i}\right)
$$

for every $x \in H$. Since $f$ is continuous and $H$ is dense in $G$, it follows that (4) holds for every $x \in G$. Thus $T_{h} f \in V$ for every $h \in H$.

Recall that $C(G, E)$, the set of continuous functions mapping $G$ into $E$, endowed with the topology of uniform convergence on compact sets is a topological vector space. Then $V$ is a closed subspace of $C(G, E)$, as this is true for every finite dimensional subspace (see [13, Theorem 1.21]).

We show that $L_{f} \subset V$. Let $g \in G$ be arbitrary; we prove $T_{g} f \in V$. Since $V$ is closed, it is enough to show that for every neighbourhood $W$ of $T_{g} f$ there is a $\phi \in V \cap W$. Let $K \subset G$ be compact and $\varepsilon>0$ be such that $\phi \in W$ whenever $\left\|\phi(x)-T_{g} f(x)\right\|_{E}<\varepsilon$ for every $x \in K$. Since $f$ is continuous, there is a neighbourhood $Z$ of $g$ such that $\|f(x+h)-f(x+g)\|_{E}<\varepsilon$ for every $h \in Z$ and $x \in K$. Since $H$ is dense in $G$, we can choose such a $h \in H$. Then 
we have $T_{h} f \in V \cap W$, proving $L_{f} \subset V$. Since $V$ is of finite dimension, so is $L_{f}$.

Corollary 10. If $G=\mathbb{R}^{p}$ with the Euclidean topology and $E$ is a Banach space, then properties (i)-(iv) listed in Theorem 8 are equivalent.

Proof. $\mathbb{Q}^{p}$ is a dense subgroup of $\mathbb{R}^{p}$ with $r_{0}\left(\mathbb{Q}^{p}\right)=p$, and thus Theorem 9 applies.

Remark 11. We show that, in general, none of the implications (i) $\Longrightarrow$ (ii) $\Longrightarrow$ (iii) $\Longrightarrow$ (iv) can be reversed.

We saw already that the identity function defined on a Banach space of infinite dimension is a w-polynomial but not a polynomial.

We give another example. Let $F$ be the free Abelian group of countable rank. We represent $F$ as the set of sequences $x=\left(x_{1}, x_{2}, \ldots\right)$ such that $x_{i}$ is an integer for every $i$ and $x_{i}=0$ if $i$ is large enough. Let $F$ be endowed with the discrete topology.

Let $E$ be a Banach space of infinite dimension, and let the elements $e_{1}, e_{2}, \ldots \in E$ be linearly independent over $\mathbb{C}$. Then the function $f(x)=$ $\sum_{i=1}^{\infty} x_{i} e_{i}(x \in F)$ is a w-polynomial, since $(u \circ f)(x)=\sum_{i=1}^{\infty} u\left(e_{i}\right) x_{i}$ is additive, hence a polynomial on $F$ for every $u \in E^{*}$. On the other hand, $f$ is not a polynomial, as $R(f)$ is of infinite dimension.

Clearly, a complex valued function is a w-polynomial if and only if it is a polynomial. Now it is known that the function $f(x)=\sum_{i=1}^{\infty} x_{i}^{2}(x \in F)$ is a complex valued generalized polynomial, but not a polynomial, hence not a w-polynomial. In fact, it is easy to see that $f$ is a generalized polynomial of degree 2. On the other hand, $f$ is not a polynomial, as $\operatorname{dim} L_{f}=\infty$ (see [16]).

Finally, $P(x)=\sum_{1}^{\infty} x_{i}^{i}(x \in F)$ is a complex valued local polynomial, but not a generalized polynomial (see [7, Proposition 1]).

We say that a continuous function $f \in C(G, E)$ is a w-exponential polynomial, if $u \circ f$ is a (complex valued) exponential polynomial for every $u \in E^{*}$.

Lemma 12. If $f \in C(G, E)$ is a w-exponential polynomial (in particular, if $f$ is a w-polynomial), then there exists a positive integer $N$ such that $\operatorname{dim} L_{u \circ f} \leq N$ for every $u \in E^{*}$.

Proof. It is easy to see that

$$
L_{u \circ f}=\left\{u \circ \phi: \phi \in L_{f}\right\}
$$

for every $f \in E^{G}$ and $u \in E^{*}$. If $f \in C(G, E)$ is a w-exponential polynomial and $u \in E^{*}$, then $u \circ f$ is a complex valued exponential polynomial, and thus $\operatorname{dim} L_{u \circ f}$ is finite. For every positive integer $n$, let $E_{n}^{*}$ be the set of linear functionals $u \in E^{*}$ such that $\operatorname{dim} L_{u \circ f}<n$. Then we have $E^{*}=\bigcup_{n=1}^{\infty} E_{n}^{*}$.

We prove that $E_{n}^{*}$ is closed. Clearly, $u \in E_{n}^{*}$ if and only if, for every $f_{1}, \ldots, f_{n} \in L_{f}$, the complex valued functions $u \circ f_{1}, \ldots, u \circ f_{n}$ are linearly dependent over $\mathbb{C}$. This is true if and only if the determinant 
$\operatorname{det}\left|u\left(f_{i}\left(x_{j}\right)\right)\right|_{i, j=1, \ldots, n}$ is zero for every $x_{1}, \ldots, x_{n}$ (see [1, Lemma 1, p. 229]). It is easy to see that for every $f_{1}, \ldots, f_{n} \in L_{f}$ and $x_{1}, \ldots, x_{n} \in G$ the set of linear functionals $u \in E^{*}$ such that det $\left|u\left(f_{i}\left(x_{j}\right)\right)\right|_{i, j=1, \ldots, n}=0$ is closed. Thus $E_{n}^{*}$, the intersection of these closed sets, is also closed.

The Baire category theorem implies that there is an $n$ with int $E_{n}^{*} \neq \emptyset$. Suppose $B\left(u_{0}, r\right) \subset E_{n}^{*}$, where $B\left(u_{0}, r\right)$ is the ball with center $u_{0}$ and radius $r$.

Let $u \in E^{*}$ be arbitrary. Then there is a $\lambda \in \mathbb{C} \backslash\{0\}$ such that $u_{0}+\lambda u \in$ $B\left(u_{0}, r\right) \subset E_{n}^{*}$. By (5), the linear space $\left\{u_{0} \circ \phi+\lambda \cdot u \circ \phi: \phi \in L_{f}\right\}$ equals $L_{\left(u_{0}+\lambda u\right) \circ f}$, hence is of dimension $<n$. Since the linear space $\left\{u_{0} \circ \phi: \phi \in\right.$ $\left.L_{f}\right\}=L_{u_{0} \circ f}$ is also of dimension $<n$, it follows that the dimension of $L_{u \circ f}=$ $\left\{u \circ \phi: \phi \in L_{f}\right\}$ is less than $2 n$.

Remark 13. It is easy to prove that if $f: G \rightarrow \mathbb{C}$ is a complex valued polynomial, then $\operatorname{deg} f<\operatorname{dim} L_{f}$ (see [8, Proposition 4]). If $f \in C(G, E)$ is a w-polynomial, then $\operatorname{dim} L_{f}$ can be infinite (see Remark 11). However, by the previous lemma, there is a smallest integer $N(f)$ such that $\operatorname{dim} L_{u \circ f} \leq N(f)$ for every $u \in E^{*}$.

By Theorem 8, every w-polynomial is a generalized polynomial, and thus has a degree. We show that $\operatorname{deg} f<N(f)$ holds for every w-polynomial $f$.

If $u \in E^{*}$, then $u \circ f$ is a polynomial, therefore, a generalized polynomial. Then, by (iii) of Theorem 1 , there is an $u_{0} \in E^{*} \operatorname{such}$ that $\operatorname{deg}\left(u_{0} \circ f\right)=\operatorname{deg} f$. Since $u_{0} \circ f$ is a complex valued polynomial, we obtain

$$
\operatorname{deg} f=\operatorname{deg}\left(u_{0} \circ f\right)<\operatorname{dim} L_{u_{0} \circ f} \leq N(f) .
$$

We also note that $N(f)$ is not bounded from above by any function of $\operatorname{deg} f$. If, for example, $G=\mathbb{R}^{n}$ and $f=x_{1}^{2}+\ldots+x_{n}^{2}$, then $\operatorname{deg} f=2$. On the other hand, $L_{f}$ is generated by the functions $f, x_{i}(i=1, \ldots, n)$ and the constants, and thus $\operatorname{dim} L_{f}=n+2$.

Our next aim is to prove the following description of w-exponential polynomials.

Theorem 14. A function $f \in C(G, E)$ is a w-exponential polynomial if and only if there are finitely many $w$-polynomials $p_{1}, \ldots, p_{n} \in C(G, E)$ and complex valued exponentials $m_{1}, \ldots, m_{n} \in \mathbb{C}^{G}$ such that $f=m_{1} p_{1}+\ldots+m_{n} p_{n}$.

An operator $D: E^{G} \rightarrow E^{G}$ is called a difference operator, if $D$ is the linear combination with complex coefficients of finitely many translation operators. Note that $\Delta_{g}=T_{g}-T_{0}$ is also a difference operator.

Lemma 15. For every finite set of distinct exponentials $\left\{m_{1}, \ldots, m_{n}\right\}$ and for every integer $s \geq-n$ there exists a finite set $\mathcal{D}$ of difference operators with the following property: whenever $p_{1}, \ldots, p_{n}$ are complex valued generalized polynomials on $G$ such that $\sum_{i=1}^{n} \operatorname{deg} p_{i} \leq s$ and $f=\sum_{i=1}^{n} p_{i} \cdot m_{i}$, then for every $1 \leq i \leq n$ there is a $D \in \mathcal{D}$ with $p_{i} \cdot m_{i}=D f$. 
Proof. If $n=1$, then $\mathcal{D}=\left\{T_{0}\right\}$ works, independently of $s$. Therefore, we may assume $n>1$.

We prove by induction on $s$. Note that, by definition, the degree of the identically zero function is -1 . If $s=-n$, then $p_{i}=0$ for every $i$, and thus $\mathcal{D}=\{\boldsymbol{0}\}$ works, where $\mathbf{0}$ denotes the identically zero operator (which maps every function to the identically zero function).

Suppose that $s>-n$, and that the statement is true for the smaller values. Let $\mathcal{D}$ be a finite set of difference operators such that whenever $q_{1}, \ldots, q_{n}$ are generalized polynomials with $\sum_{i=1}^{n} \operatorname{deg} q_{i} \leq s-1$ and $f_{1}=\sum_{i=1}^{n} q_{i} m_{i}$, then for every $i$ there is a $D \in \mathcal{D}$ such that $D f_{1}=q_{i} m_{i}$. We may assume that $\mathbf{0} \in \mathcal{D}$.

From $s>-n$ it follows that $\max _{1 \leq i \leq n} \operatorname{deg} p_{i} \geq 0$. We may assume that $\operatorname{deg} p_{1} \geq 0$. Since $m_{1} \neq m_{n}$ by assumption, we can fix an element $g \in G$ such that $m_{1}(g) \neq m_{n}(g)$. We put $D_{0}=T_{g}-m_{1}(g) \cdot T_{0}$. Then

$$
D_{0}(p \cdot m)=T_{g} p \cdot T_{g} m-m_{1}(g) \cdot p \cdot m=\left(m(g) \cdot T_{g} p-m_{1}(g) \cdot p\right) \cdot m
$$

for every $p \in \mathbb{C}^{G}$ and exponential $m$. Therefore, we have

$$
D_{0} f=m_{1}(g) \cdot \Delta_{g} p_{1} \cdot m_{1}+\sum_{i=2}^{n}\left(\left(m_{i}(g) \cdot T_{g} p_{i}-m_{1}(g) \cdot p_{i}\right) \cdot m_{i} .\right.
$$

Since $\operatorname{deg} \Delta_{g} p_{1}<\operatorname{deg} p_{1}$ and $\operatorname{deg}\left(\left(m_{i}(g) \cdot T_{g} p_{i}-m_{1}(g) \cdot p_{i}\right) \leq \operatorname{deg} p_{i}\right.$, it follows from the choice of $\mathcal{D}$ that $m_{1}(g) \cdot \Delta_{g} p_{1} \cdot m_{1}=D_{1} D_{0} f$ for some $D_{1} \in \mathcal{D}$. Similarly, for every $i$ such that $p_{i} \neq 0$ there is a $D_{i} \in \mathcal{D}$ with $m_{i}(g) \Delta_{g} p_{i} \cdot m_{i}=$ $D_{i} D_{0} f$. If $p_{i}=0$ then we can take $D_{i}=\mathbf{0}$, and thus there is such a $D_{i}$ in both cases. By (6) and by the choice of $\mathcal{D}$ we have

$$
\left(m_{n}(g) \cdot T_{g} p_{n}-m_{1}(g) \cdot p_{n}\right) \cdot m_{n}=E_{n} D_{0} f
$$

with a suitable $E_{n} \in \mathcal{D}$. Since

$$
\begin{aligned}
& \left(m_{n}(g) \cdot T_{g} p_{n}-m_{1}(g) \cdot p_{n}\right) \cdot m_{n}= \\
& \quad=m_{n}(g) \cdot \Delta_{g} p_{n} \cdot m_{n}+\left(m_{n}(g)-m_{1}(g)\right) \cdot p_{n} \cdot m_{n}
\end{aligned}
$$

and $m_{n}(g) \cdot \Delta_{g} p_{n} \cdot m_{n}=D_{n} D_{0} f$, we obtain

$$
E_{n} D_{0} f=D_{n} D_{0} f+\left(m_{n}(g)-m_{1}(g)\right) \cdot p_{n} \cdot m_{n}
$$

and

$$
p_{n} \cdot m_{n}=c \cdot E_{n} D_{0} f-c \cdot D_{n} D_{0} f,
$$

where $c=1 /\left(m_{n}(g)-m_{1}(g)\right)$. Therefore, if we add the operators $c D D_{0}-c D^{\prime} D_{0}$ $\left(D, D^{\prime} \in \mathcal{D}\right)$ to $\mathcal{D}$, then $p_{n} \cdot m_{n}=E f$ will hold for a suitable $E$ belonging to the enlarged $\mathcal{D}$. (Note that the element $g$ does not depend on the functions $p_{1}, \ldots, p_{n}$, only on $m_{1}$ and $m_{n}$.) The same argument provides finitely many operators such that if we add them to $\mathcal{D}$ then, for every $i=1, \ldots, n, p_{i} m_{i}=E f$ will hold for a suitable $E$ belonging to the enlarged $\mathcal{D}$. 
Proof of Theorem 14. Suppose $f=m_{1} p_{1}+\ldots+m_{n} p_{n}$, where $p_{1}, \ldots, p_{n}$ are w-polynomials and $m_{1}, \ldots, m_{n}$ are complex valued exponentials. If $u \in E^{*}$, then $u \circ f=m_{1} \cdot u \circ p_{1}+\ldots+m_{n} \cdot u \circ p_{n}$. Since $p_{i}$ is a w-polynomial, it follows that $u \circ p_{i}$ is a complex valued polynomial for every $i=1, \ldots, n$, and thus $u \circ f$ is an exponential polynomial. This is true for every $u \in E^{*}$, proving that $f$ is a w-exponential polynomial.

Now suppose that $f$ is a w-exponential polynomial. By Lemma 12 , there is a positive integer $K$ such that $\operatorname{dim} L_{u \circ f}<K$ for every $u \in E^{*}$.

Let $\mathcal{P}$ denote the set of all functions $p \cdot m$ such that $p: G \rightarrow \mathbb{C}$ is a polynomial and $m: G \rightarrow \mathbb{C}$ is an exponential. If $u \in E^{*}$, then $u \circ f$ is an exponential polynomial, and thus it is the sum of finitely many elements of $\mathcal{P}$. In other words, for every $u \in E^{*}$ there exists a finite set $\mathcal{P}_{u} \subset \mathcal{P}$ such that $u \circ f=\sum_{p \cdot m \in \mathcal{P}_{u}} p \cdot m$.

Let $\mathcal{M}$ denote the set of those exponentials $m$ for which there exist $u \in E^{*}$ and a nonzero polynomial $p$ such that $p \cdot m \in \mathcal{P}_{u}$. We prove that $\mathcal{M}$ contains less than $K$ distinct exponentials.

Suppose this is not true, and let $m_{1}, \ldots, m_{K}$ be distinct exponentials in $\mathcal{M}$. We may assume that for every $u \in E^{*}$ and $1 \leq i \leq K$ there is a unique polynomial $p_{u, i}$ such that $p_{u, i} \cdot m_{i} \in \mathcal{P}_{u}$. Indeed, if $\mathcal{P}_{u}$ does not contain such a product, then we add $0 \cdot m_{i}$ to $\mathcal{P}_{u}$, and put $p_{u, i}=0$.

For every $1 \leq i \leq K$ we have $m_{i} \in \mathcal{M}$, and thus there is an $u_{i} \in E^{*}$ such that $p_{u_{i}, i} \neq 0$. We show that there are complex numbers $\lambda_{1}, \ldots, \lambda_{K}$ such that

$$
\sum_{i=1}^{K} \lambda_{i} p_{u_{i}, j} \neq 0
$$

for every $j=1, \ldots, K$. Indeed, for a fixed $j$, the set of $K$-tuples $\left(\lambda_{1}, \ldots, \lambda_{K}\right)$ such that $\sum_{i=1}^{K} \lambda_{i} p_{u_{i}, j}=0$ is a linear subspace $L_{j}$ of $\mathbb{C}^{K}$. Since $p_{u_{j}, j} \neq 0$, the subspace $L_{j}$ does not contain the vector $(0, \ldots, 0,1,0, \ldots, 0)$ having 1 as the $j$ th coordinate. Therefore, $L_{j}$ is a proper subspace of $\mathbb{C}^{K}$. Now $\mathbb{C}^{K}$ is not the union of finitely many proper subspaces, therefore, we must have (7) for every $j=1, \ldots, K$ with a suitable $\left(\lambda_{1}, \ldots, \lambda_{K}\right)$.

Let $u=\sum_{i=1}^{K} \lambda_{i} u_{i}$. Then $p_{u, j}=\sum_{i=1}^{K} \lambda_{i} p_{u_{i}, j} \neq 0$ for every $j=1, \ldots, K$. That is, in the representation of $u \circ f$ as a sum of functions $p \cdot m \in \mathcal{P}$, each of $m_{1}, \ldots, m_{K}$ appears with a nonzero polynomial factor.

Now we need the following result: if $V$ is a translation invariant linear subspace of $\mathbb{C}^{G}$ and $\sum_{i=1}^{n} p_{i} m_{i} \in V$, where $p_{i} \in \mathbb{C}^{G}$ is a nonzero generalized polynomial for every $i=1, \ldots, n$ and $m_{1}, \ldots, m_{n}$ are distinct complex valued exponentials, then $m_{i} \in V$ for every $i=1, \ldots, n$. This is proved, e.g., in [7, Lemma 6] in the case when $G$ is an Abelian group. One can easily check that the same proof works in Abelian semigroups.

Since $L_{u \circ f}$ is a translation invariant linear subspace of $\mathbb{C}^{G}$ and $u \circ f=$ $\sum_{p m \in \mathcal{P}_{u}} p \cdot m$, it follows that $m_{1}, \ldots, m_{K} \in L_{u \circ f}$. Now $m_{1}, \ldots, m_{K}$ are linearly independent over $\mathbb{C}$, since, if $\sum_{i=1}^{K} c_{i} m_{i}=0$, where $c_{1}, \ldots, c_{K} \in \mathbb{C}$, then the 
unique representation of the zero function implies $c_{1}=c_{2}=\ldots=c_{K}=0$. We find that $\operatorname{dim} L_{u \circ f} \geq K$, which contradicts the choice of $K$.

This contradiction proves that $\mathcal{M}$ contains less than $K$ exponentials. Let $\mathcal{M}=\left\{m_{1}, \ldots, m_{n}\right\}$, where $n<K$. Then for every $u \in E^{*}$ there are polynomials $p_{u, i}(i=1, \ldots, n)$ such that $u \circ f=\sum_{i=1}^{n} p_{u, i} \cdot m_{i}$.

Let $s(u)=\max _{1 \leq i \leq n} \operatorname{deg} p_{u, i}$, and put $U_{s}=\left\{u \in E^{*}: s(u) \leq s\right\}$ for every positive integer $s$. By the Baire category theorem we can find an integer $s$ such that $U_{s}$ is of second category in $E^{*}$.

By Lemma 15, there is a finite set $\mathcal{D}$ of difference operators such that, for every $u \in U_{s}$ and $i=1, \ldots, n, p_{u, i} \cdot m_{i}=D_{i}(u \circ f)$ for some $D_{i} \in \mathcal{D}$. Since $\mathcal{D}$ is finite, there is an $n$-tuple $\left(D_{1}, \ldots, D_{n}\right)$ such that the set $B$ of linear functionals $u \in U_{s}$ such that

$$
p_{u, i} \cdot m_{i}=D_{i}(u \circ f) \quad(i=1, \ldots, n)
$$

is also of second category in $E^{*}$. We fix such an $n$-tuple $\left(D_{1}, \ldots, D_{n}\right)$.

We show that $B$ is a closed linear subspace of $E^{*}$. Suppose $u_{1}, u_{2} \in B$, $\lambda_{1}, \lambda_{2} \in \mathbb{C}$, and put $u=\lambda_{1} u_{1}+\lambda_{2} u_{2}$. Since $u_{j} \circ f=\sum_{i=1}^{n} p_{u_{j}, i} \cdot m_{i}(j=1,2)$, we have

$$
u \circ f=\sum_{i=1}^{n}\left(\lambda_{1} p_{u_{1}, i}+\lambda_{2} p_{u_{2}, i}\right) \cdot m_{i} .
$$

The uniqueness of the representation gives

$$
p_{u, i}=\lambda_{1} p_{u_{1}, i}+\lambda_{2} p_{u_{2}, i}
$$

and

$$
\begin{aligned}
D_{i}(u \circ f) & =\lambda_{1} D_{i}\left(u_{1} \circ f\right)+\lambda_{2} D_{i}\left(u_{2} \circ f\right)= \\
& =\lambda_{1} p_{u_{1}, i} \cdot m_{i}+\lambda_{2} p_{u_{2}, i} \cdot m_{i}= \\
& =p_{u, i} \cdot m_{i} .
\end{aligned}
$$

Thus $u \in B$, proving that $B$ is a linear subspace of $E^{*}$. Let $u \in E^{*}$ be in the closure of $B$. Then there is a sequence of linear functionals $u_{\nu} \in B$ such that $\left\|u_{\nu}-u\right\| \rightarrow 0$. Then

$$
p_{u_{\nu}, i} \cdot m_{i}=D_{i}\left(u_{\nu} \circ f\right) \rightarrow D_{i}(u \circ f)
$$

as $\nu \rightarrow \infty$, for every $i=1, \ldots, n$. Thus $p_{u_{\nu}, i} \rightarrow q_{i}$ pointwise, where $q_{i}=$ $D_{i}(u \circ f) / m_{i}$.

Now $u_{\nu} \in B \subset U_{s}$, and thus $p_{u_{\nu}, i}$ is a generalized polynomial of degree $\leq s$. It is easy to check that this property is preserved under pointwise convergence in the discrete topology of $G$. Therefore, $q_{i}$ is generalized polynomial of degree $\leq s$ for every $i=1, \ldots, n$ in the discrete topology of $G$. Now we have

$$
\sum_{i=1}^{n} p_{u, i} \cdot m_{i}=u \circ f=\lim _{\nu \rightarrow \infty} u_{\nu} \circ f=\lim _{\nu \rightarrow \infty} \sum_{i=1}^{n} p_{u_{\nu}, i} \cdot m_{i}=\sum_{i=1}^{n} q_{i} \cdot m_{i} .
$$


Then the uniqueness of the representation gives $q_{i}=p_{u, i}$ for every $i=1, \ldots, n$. Thus $p_{u, i}=D_{i}(u \circ f) / m_{i}, D_{i}(u \circ f)=p_{u, i} \cdot m_{i}$ for every $i=1, \ldots, n$; that is $u \in B$.

Thus $B$ is a closed subspace of $E^{*}$. Since $B$ is of second category, we have $B=E^{*}$. Therefore, (8) holds for every $u \in E^{*}$.

Let $p_{i}=\left(D_{i} f\right) / m_{i}(i=1, \ldots, n)$ and $\bar{f}=\sum_{i=1}^{n} p_{i} \cdot m_{i}$. Since

$$
u \circ p_{i}=u \circ\left(D_{i} f\right) / m_{i}=D_{i}(u \circ f) / m_{i}=p_{u, i}
$$

is a polynomial for every $u \in E^{*}$, it follows that $p_{i}$ is a w-polynomial for every $i=1, \ldots, n$. Now

$$
\begin{aligned}
u \circ \bar{f} & =\sum_{i=1}^{n}\left(u \circ p_{i}\right) \cdot m_{i}=\sum_{i=1}^{n} u \circ D_{i} f= \\
& =\sum_{i=1}^{n} D_{i}(u \circ f)=\sum_{i=1}^{n} p_{u, i} \cdot m_{i}= \\
& =u \circ f
\end{aligned}
$$

for every $u \in E^{*}$. Thus $\bar{f}=f$, which completes the proof.

The following result is an immediate consequence of Theorems 7,9 and 14 .

Corollary 16. Let $G$ be a topological Abelian group, and suppose that there is a dense subgroup $H$ of $G$ such that $r_{0}(H)<\infty$. Then a function $f \in C(G, E)$ is a w-exponential polynomial if and only if $f$ is an exponential polynomial.

In particular, if $G=\mathbb{R}^{p}$ with the Euclidean topology and $E$ is a Banach space, then a function $f \in C(G, E)$ is a w-exponential polynomial if and only if $f$ is an exponential polynomial.

\section{Vector Valued Harmonic Analysis and Synthesis on Discrete Abelian Groups}

Let $G$ be a topological Abelian group, and $E$ be a Banach space. We denote by $C(G, E)$ the set of continuous functions $f: G \rightarrow E$. We equip $C(G, E)$ with the topology of uniform convergence on compact sets. In this topology a set $U \subset C(G, E)$ is open if, for every $f \in U$, there exists a compact set $K \subset G$ and there is an $\varepsilon>0$ such that $\phi \in U$ whenever $\phi \in C(G, E)$ is such that $\|\phi(x)-f(x)\|_{E}<\varepsilon$ for every $x \in K$. This topology makes $C(G, E)$ a locally convex topological vector space over the complex field.

Translation invariant closed linear subspaces of $C(G, E)$ are called varieties. If $f \in C(G, E)$, then $V_{f}$ denotes the smallest variety containing $f$. Clearly, $V_{f}$ equals the closure of $L_{f}$. We say that spectral synthesis holds in $C(G, E)$ if every variety $V$ in $C(G, E)$ is the closed linear hull of the set of exponential polynomials contained in $V$. 
It is known that spectral synthesis holds in $C(G, E)$ for every compact Abelian group $G$ and for every Banach space $E$. In fact, this result follows from the approximation theorem of almost periodic functions mapping a group into a Banach space [2]. More precisely, if $G$ is a compact Abelian group, then (i) every continuous function $f: G \rightarrow E$ is almost periodic, (ii) the terms of the Fourier series of $f$ are constant multiples of characters belonging to $V_{f}$, and (iii) it follows from the approximation theorem that linear combinations of these characters approximate $f$ uniformly. We note, however, that this special case can be proved without the machinery of almost periodic functions, and in the next section we provide a simple self-contained proof.

As it turns out, the situation for locally compact Abelian groups is different. In the rest of this section we concentrate on discrete Abelian groups. In these groups we have $C(G, E)=E^{G}$. As we will see shortly, spectral synthesis does not hold in $E^{G}$ if $G$ is infinite and $E$ is of infinite dimension. Actually, the situation is even worse.

By a generalized (resp. local) exponential polynomial we mean a function of the form $\sum_{i=1}^{n} m_{i} \cdot p_{i}$, where $m_{i}$ is an exponential and $p_{i} \in E^{G}$ is a generalized (resp. local) polynomial on $G$ for every $i=1, \ldots, n$. We say that generalized (resp. local) spectral synthesis holds in $E^{G}$ if every variety $V$ in $E^{G}$ is the closed linear hull of the set of generalized (resp. local) exponential polynomials contained in $V$. Clearly, the condition that spectral synthesis holds in $E^{G}$ implies that generalized spectral synthesis holds in $E^{G}$, and this condition, in turn, implies that local spectral synthesis holds in $E^{G}$.

Let $f=\sum_{i=1}^{n} m_{i} \cdot p_{i}$, where $m_{1}, \ldots, m_{n}$ are distinct exponentials and $p_{1}, \ldots, p_{n}$ are nonzero local polynomials on $G$. One can prove that $m_{i} \cdot p_{i} \in V_{f}$ for every $i$, moreover, there are nonzero elements $e_{1}, \ldots, e_{n} \in E$ such that $m_{i} \cdot e_{i} \in V_{f}$ for every $i$. (See [7, Lemma 7], where the complex valued case is proved. One can easily check that the proof works in the general case as well.) This result shows that if local spectral synthesis holds in $E^{G}$, then spectral analysis holds in $E^{G}$; that is, every nonzero variety in $E^{G}$ contains a function of the form $m \cdot e$, where $m$ is an exponential and $e \in E, e \neq 0$.

Theorem 17. If $G$ is an infinite discrete Abelian group and $E$ is a Banach space of infinite dimension, then spectral analysis does not hold in $E^{G}$.

Proof. It is easy to see that if spectral analysis holds in $E^{G}$, then the same is true in $E^{H}$ for every subgroup $H$ of $G$. (For the complex case see [10, Lemma 4]. The proof in the general case is the same.) Therefore, in order to prove the theorem, it is enough to find a subgroup $H$ of $G$ such that spectral analysis does not hold in $E^{H}$.

If $G$ contains an element $h$ of infinite order, then we let $H$ be the cyclic group generated by $h$. If $G$ is torsion, then we choose a countably infinite subset $A \subset G$, and let $H$ be the subgroup generated by $A$. Then $H$ is countably infinite, and is either cyclic, or torsion. 
Let $g_{1}, g_{2}, \ldots$ be an enumeration of the elements of $H$. If $H$ is cyclic generated by the element $h$, then we choose an enumeration such that $g_{2 n}=h^{n}$ $(n=1,2, \ldots)$. If $H$ is torsion, then the enumeration can be arbitrary.

Since $E$ is of infinite dimension, it contains a basic sequence $\left(x_{n}\right)$ (see $[4$, Corollary 3, p. 39]). We may assume that $\left\|x_{n}\right\|=n$ ! for every $n=1,2, \ldots$

We define $f\left(g_{n}\right)=x_{n}$ for every $n=1,2, \ldots$, and prove that $V_{f}$ does not contain any function of the form $m \cdot e$, where $m$ is an exponential and $e \in E$, $e \neq 0$.

Suppose this is false, and let $m \cdot e \in V_{f}$, where $m$ and $e$ are as above. Since $H$ is countable and $m \cdot e \in V_{f}=\mathrm{cl} L_{f}$, it follows that there is a sequence of functions $f_{k} \in L_{f}$ such that $f_{k} \rightarrow m \cdot e$ pointwise on $H$. Now each $f_{k}$ is a linear combination of the functions $T_{g_{n}} f$, and thus there is a sequence of positive integers $s_{1}<s_{2}<\ldots$ such that $f_{k}$ is a linear combination of the functions $T_{g_{n}} f\left(n=1, \ldots, s_{k}\right)$. Let

$$
f_{k}=\sum_{n=1}^{s_{k}} c_{k n} T_{g_{n}} f \quad(k=1,2, \ldots) .
$$

Let $e=\sum_{n=1}^{\infty} \alpha_{n} x_{n}$ with suitable complex coefficients $\alpha_{n}$. Since the series converges in norm, it follows that $\left\|\alpha_{n} x_{n}\right\| \rightarrow 0$, that is, $n !\left|\alpha_{n}\right| \rightarrow 0$. We have

$$
\sum_{n=1}^{s_{k}} c_{k n} x_{n}=\sum_{n=1}^{s_{k}} c_{k n} f\left(g_{n}\right)=f_{k}(0) \rightarrow m(0) \cdot e=e=\sum_{n=1}^{\infty} \alpha_{n} x_{n}
$$

as $k \rightarrow \infty$. Since the coefficient functionals are continuous (see [4, p. 32]), it follows that $\lim _{k \rightarrow \infty} c_{k n}=\alpha_{n}$ for every $n$. Let the indices $i$ and $j$ be given, and put $g=g_{j}-g_{i}$, Then

$$
f_{k}(g)=\sum_{n=1}^{s_{k}} c_{k n} f\left(g+g_{n}\right) \rightarrow m(g) \cdot e=\sum_{n=1}^{\infty}\left(m(g) \alpha_{n}\right) x_{n}
$$

as $k \rightarrow \infty$. The elements $g+g_{n}\left(n=1, \ldots, s_{k}\right)$ are distinct, and then so are $f\left(g+g_{n}\right)$. If $k$ is large enough, then $i<s_{k}, f\left(g+g_{i}\right)=f\left(g_{j}\right)=x_{j}$, and thus $f_{k}(g)$ is a linear combination of finitely many of the elements $x_{n}$ including $x_{j}$. Since the coefficient functionals are continuous, it follows that the coefficient of $x_{j}$ converges to $m(g) \alpha_{j}$ as $k \rightarrow \infty$. That is, we have $\lim _{k \rightarrow \infty} c_{k i}=m(g) \alpha_{j}$. However, as $c_{k i} \rightarrow \alpha_{i}$, we find

$$
\alpha_{i}=m(g) \alpha_{j}=m\left(g_{j}-g_{i}\right) \alpha_{j}=\left(m\left(g_{j}\right) / m\left(g_{i}\right)\right) \alpha_{j}
$$

and $\alpha_{i} m\left(g_{i}\right)=\alpha_{j} m\left(g_{j}\right)$. This is true for every $i$ and $j$, and thus there is a complex number $c$ such that $\alpha_{i} m\left(g_{i}\right)=c$ for every $i=1,2, \ldots$

Since $e \neq 0$, we have $\alpha_{n} \neq 0$ for at least one $n$, and thus $c \neq 0$. Now we consider the two cases concerning the group structure of $H$. If $H$ is torsion, then the value of $m(g)$ is a root of unity for every $g \in H$. Then $\left|\alpha_{n}\right|=\left|c / m\left(g_{n}\right)\right|=|c|$ for every $n$. This, however, is impossible by $n !\left|\alpha_{n}\right| \rightarrow 0$. 
Next suppose that $H$ is cyclic with generator $h$. Then we have $g_{2 n}=h^{n}$ for every $n=1,2, \ldots$. Let $m(h)=\lambda$, then $m\left(g_{2 n}\right)=\lambda^{n}$ and $\left|\alpha_{2 n}\right|=$ $\left|c / m\left(g_{2 n}\right)\right|=|c| \cdot|\lambda|^{-n}$ for every $n$. This, again, contradicts $n !\left|\alpha_{n}\right| \rightarrow 0$, completing the proof.

Returning to the question of spectral synthesis in discrete Abelian groups, the previous result shows that spectral synthesis can hold in $E^{G}$ only if $G$ is finite or $E$ is of finite dimension. If $G$ is finite, then every element $f \in E^{G}$ is an exponential polynomial, as $L_{f}$ is of finite dimension. Therefore, in this case spectral synthesis does hold.

If $E$ is of finite dimension, then spectral synthesis in $E^{G}$ is still not automatic. Indeed, if spectral synthesis holds in $E^{G}$, then it also holds in $\mathbb{C}^{G}$. Now it is known that spectral synthesis holds in $\mathbb{C}^{G}$ if and only if $r_{0}(G)$ (the torsion free rank of $G$ ) is finite (see [11, Theorem 1]). So the only cases left are when $r_{0}(G)$ is finite and $E$ is of finite dimension. In the next theorem we show that spectral synthesis does hold in these cases.

We also consider local spectral synthesis and spectral analysis. Note that spectral analysis holds in $\mathbb{C}^{G}$ if and only if $r_{0}(G)$ is less than continuum (see [10, Theorem 1]). Also, there exists an uncountable cardinal $\kappa$ such that local spectral synthesis holds in $\mathbb{C}^{G}$ if and only if $r_{0}(G)<\kappa$ (see [7, Theorem 3]). In particular, local spectral synthesis holds in $\mathbb{C}^{G}$ for every countable discrete Abelian group.

Theorem 18. Let $G$ be a discrete Abelian group, and let $k$ be a positive integer.

(i) If $r_{0}(G)$ is finite, then spectral synthesis holds in $\left(\mathbb{C}^{k}\right)^{G}$.

(ii) If $r_{0}(G)<\kappa$, then local spectral synthesis holds in $\left(\mathbb{C}^{k}\right)^{G}$.

For every set $V$ of maps $f: G \rightarrow \mathbb{C}^{k}$ we shall denote by $\bar{V}$ the set of maps

$$
\mathbb{Z}^{k} \times G \ni\left(t_{1}, \ldots, t_{k}, x\right) \mapsto t_{1} f_{1}(x)+\ldots+t_{k} f_{k}(x)+g(x),
$$

where $\left(f_{1}, \ldots, f_{k}\right) \in V$ and $g: G \rightarrow \mathbb{C}$.

Lemma 19. If $V$ is a variety of maps $f: G \rightarrow \mathbb{C}^{k}$, then $\bar{V}$ is a variety on $\mathbb{Z}^{k} \times G$.

Proof. Suppose that $V$ is a variety. It is clear that $\bar{V}$ is a translation invariant linear space. We show that $\bar{V}$ is closed.

Let $e_{i}=\left(\delta_{1 i}, \ldots, \delta_{k i}, 0\right) \in \mathbb{Z}^{k} \times G$ for every $i=1, \ldots, k$, where $\delta_{j i}$ is the Kronecker delta. It is clear that if the function $F$ is defined by (9), then $\Delta_{e_{i}} F\left(t_{1}, \ldots, t_{k}, x\right)=f_{i}(x)$ for every $i=1, \ldots, k$ and $\left(t_{1}, \ldots, t_{k}, x\right) \in \mathbb{Z}^{k} \times G$. Suppose that $h:\left(\mathbb{Z}^{k} \times G\right) \rightarrow \mathbb{C}$ is in the closure of $\bar{V}$. From the previous observation it follows that $\Delta_{e_{i}} h$ does not depend on the variables $t_{1}, \ldots, t_{k}$. That is, there are functions $h_{1}, \ldots, h_{k}: G \rightarrow \mathbb{C}$ such that $\Delta_{e_{i}} h\left(t_{1}, \ldots, t_{k}, x\right)=$ $h_{i}(x)$ for every $i=1, \ldots, k$ and $\left(t_{1}, \ldots, t_{k}, x\right) \in \mathbb{Z}^{k} \times G$. Let

$$
s\left(t_{1}, \ldots, t_{k}, x\right)=t_{1} h_{1}(x)+\ldots+t_{k} h_{k}(x)
$$


for every $\left(t_{1}, \ldots, t_{k}, x\right) \in \mathbb{Z}^{k} \times G$, and put $g=h-s$. Then $\Delta_{e_{i}} g=0$ for every $i=1, \ldots, k$. Thus $g=h-s$ does not depend on the variables $t_{1}, \ldots, t_{k}$. Therefore, we have

$$
h\left(t_{1}, \ldots, t_{k}, x\right)=t_{1} h_{1}(x)+\ldots+t_{k} h_{k}(x)+g(x)
$$

for every $\left(t_{1}, \ldots, t_{k}, x\right) \in \mathbb{Z}^{k} \times G$. We prove that $\left(h_{1}, \ldots, h_{k}\right) \in V$. Since $V$ is a variety, it is enough to show that $\left(h_{1}, \ldots, h_{k}\right)$ is in the closure of $V$.

Let the finite set $X \subset G$ and the positive number $\varepsilon$ be given. Since $h$ is in the closure of $\bar{V}$, there is a function $f \in \bar{V}$ such that it is closer to $h$ than $\varepsilon / 2$ at each point $\left(t_{1}, \ldots, t_{k}, x\right)$, where $t_{i}=0,1$ for every $i=1, \ldots, k$ and $x \in X$. Let

$$
f\left(t_{1}, \ldots, t_{k}, x\right)=t_{1} f_{1}(x)+\ldots+t_{k} f_{k}(x)+g_{1}(x),
$$

where $\left(f_{1}, \ldots, f_{k}\right) \in V$. Since $\Delta_{e_{i}} h(0, \ldots, 0, x)=h_{i}(x)$ and $\Delta_{e_{i}} f(0, \ldots, 0, x)=$ $f_{i}(x)$ for every $x \in X$, it follows that $\left|h_{i}(x)-f_{i}(x)\right|<\varepsilon$ for every $x \in X$ and $i=1, \ldots, k$. This proves that $\left(h_{1}, \ldots, h_{k}\right)$ is in the closure of $V$. Thus $\left(h_{1}, \ldots, h_{k}\right) \in V$ and $h \in \bar{V}$, showing that $\bar{V}$ is a variety.

Proof of Theorem 18. (i) Let $V$ be a variety of maps $f: G \rightarrow \mathbb{C}^{k}$. By Theorem 4 , a function $f: G \rightarrow \mathbb{C}^{k}$ is an exponential polynomial if and only if $f=\left(f_{1}, \ldots, f_{k}\right)$, where $f_{1}, \ldots, f_{k}$ are complex valued exponential polynomials.

We have to show that if $r_{0}(G)$ is finite, then the set of maps

$$
\left\{\left(p_{1}, \ldots, p_{k}\right) \in V: p_{1}, \ldots, p_{k} \text { are exponential polynomials }\right\}
$$

is dense in $V$. Let $\left(f_{1}, \ldots, f_{k}\right) \in V$, and let the finite set $X \subset G$ and the positive number $\varepsilon$ be given. If $r_{0}(G)$ is finite, then $r_{0}\left(\mathbb{Z}^{k} \times G\right)$ is also finite. Then, by [11, Theorem 1], spectral synthesis holds in every variety on $\mathbb{Z}^{k} \times G$. By Lemma $19, \bar{V}$ is a variety. Since the function $f=t_{1} f_{1}(x)+\ldots+t_{k} f_{k}(x)$ belongs to $\bar{V}$, it follows that there is an exponential polynomial $p \in \bar{V}$ such that $p$ is closer to $f$ than $\varepsilon / 2$ at each point $\left(t_{1}, \ldots, t_{k}, x\right)$, where $t_{i}=0,1$ for every $i=1, \ldots, k$ and $x \in X$. Let

$$
p\left(t_{1}, \ldots, t_{k}, x\right)=t_{1} p_{1}(x)+\ldots+t_{k} p_{k}(x)+g_{2}(x) .
$$

Then $\left(p_{1}, \ldots, p_{k}\right) \in V$ by $p \in \bar{V}$, and $\left|p_{i}(x)-f_{i}(x)\right|<\varepsilon$ for every $x \in X$ and $i=1, \ldots, k$. Since $p$ is an exponential polynomial, so is $p_{i}=\Delta_{e_{i}} p(0, \ldots, 0, x)$ for every $i$. This proves that the maps $\left(p_{1}, \ldots, p_{k}\right) \in V$, where $p_{1}, \ldots, p_{k}$ are exponential polynomials constitute a dense subset of $V$. This proves (i).

The proof of (ii) is similar to that of (i). If $r_{0}(G)<\kappa$, then $r_{0}\left(\mathbb{Z}^{k} \times G\right)<\kappa$. Thus local spectral synthesis holds in the variety $\bar{V}$. If $f=t_{1} f_{1}(x)+\ldots+t_{k} f_{k}(x) \in \bar{V}$, there is a local exponential polynomial $p \in \bar{V}$ such that $p$ is closer to $f$ than $\varepsilon / 2$ at each point $\left(t_{1}, \ldots, t_{k}, x\right)$, where $t_{i}=0,1$ for every $i=1, \ldots, k$ and $x \in X$. As we saw above, this implies that $\left|p_{i}(x)-f_{i}(x)\right|<\varepsilon$ for every $x \in X$ and $i=$ $1, \ldots, k$. Since $p$ is a local exponential polynomial, so is $p_{i}=\Delta_{e_{i}} p(0, \ldots, 0, x)$ 
for every $i$. This proves that the maps $\left(p_{1}, \ldots, p_{k}\right) \in V$, where $p_{1}, \ldots, p_{k}$ are local exponential polynomials constitute a dense subset of $V$.

Corollary 20. If $r_{0}(G)<\kappa$ (in particular, if $r_{0}(G)$ is countable), then spectral analysis holds in $\left(\mathbb{C}^{k}\right)^{G}$.

Proof. Let $V \subset E^{G}$ be a nonzero variety, where $E=\mathbb{C}^{k}$. By (ii) of Theorem 18, local spectral synthesis holds in $\left(\mathbb{C}^{k}\right)^{G}$, and thus there is a nonzero local exponential polynomial $f \in V$. Let $f=\sum_{i=1}^{n} m_{i} \cdot p_{i}$, where $m_{i}$ is an exponential and $p_{i} \in E^{G}$ is a local polynomial for every $i=1, \ldots, n$. We may assume that $m_{1}, \ldots, m_{n}$ are distinct and $p_{1}, \ldots, p_{n}$ are nonzero. Then, by [7, Lemma 7], $m_{i} \cdot e_{i} \in V$ for every $i=1, \ldots, n$ with nonzero $e_{1}, \ldots, e_{n} \in E=\mathbb{C}^{k}$. In fact, [7] deals with complex valued maps, but the argument of the proof of [7, Lemma 7] works for vector valued functions as well. This proves that spectral analysis holds in $V$.

Note that if $k=1$, then $\kappa$ can be replaced by $2^{\omega}$ in the statement of Corollary 20 (see [10]). Since $\omega_{1} \leq \kappa \leq 2^{\omega}$, it makes no difference under the continuum hypothesis. Still, it would be interesting to see if $\kappa$ can be replaced by $2^{\omega}$ in the cases $k>1$ as well.

Funding Open access funding provided by Eötvös Loránd University.

Open Access. This article is licensed under a Creative Commons Attribution 4.0 International License, which permits use, sharing, adaptation, distribution and reproduction in any medium or format, as long as you give appropriate credit to the original author(s) and the source, provide a link to the Creative Commons licence, and indicate if changes were made. The images or other third party material in this article are included in the article's Creative Commons licence, unless indicated otherwise in a credit line to the material. If material is not included in the article's Creative Commons licence and your intended use is not permitted by statutory regulation or exceeds the permitted use, you will need to obtain permission directly from the copyright holder. To view a copy of this licence, visit http://creativecommons.org/ licenses/by/4.0/.

\section{Appendix: A Proof of Spectral Synthesis on Compact Abelian Groups}

Theorem 21. If $G$ is a compact Abelian group and $E$ is a Banach space, then spectral synthesis holds in $C(G, E)$.

Proof. We denote by $M(G)$ the set of complex valued regular Borel measures on $G$ with finite total variation $\|\mu\|$. If $f \in C(G, E)$ and $\mu \in M(G)$, then we define $\mu * f(x)$ as $\int_{G} f(x-t) d \mu(t)$. The integral makes sense for every $x \in G$, as we integrate a continuous function mapping a compact Hausdorff space into a Banach space (see [13, Theorem 3.27]). 
We show that $\mu * f \in V_{f}$ for every $f \in C(G, E)$ and $\mu \in M(G)$. Let $\varepsilon>0$ be given. By the uniform continuity of $f$ we can find a neighbourhood $V$ of 0 such that $\|f(x)-f(y)\|_{E}<\varepsilon /\|\mu\|$ whenever $x-y \in V$. Let $U$ be a symmetric neighbourhood of 0 such that $U+U \subset V$.

Since $G$ is compact, there are points $x_{i} \in G(i=1, \ldots, n)$ such that $G=\bigcup_{i=1}^{n}\left(U+x_{i}\right)$. Choose a partition $\left\{E_{1}, \ldots, E_{n}\right\}$ of $G$ into finitely many Borel sets such that $E_{i} \subset U+x_{i}$ for every $i=1, \ldots, n$. Deleting the empty sets from the partition, we may assume that $E_{i} \neq \emptyset$ for every $i$. Choose a point $t_{i} \in E_{i}$ for every $i=1, \ldots, n$, and put

$$
z(x)=\int_{G} f(x-t) d \mu(t)-\sum_{i=1}^{n} \mu\left(E_{i}\right) f\left(x-t_{i}\right) \quad(x \in G) .
$$

Then $\|z(x)\|_{E} \leq \varepsilon$ for every $x \in G$. Indeed, suppose $\|z(x)\|_{E}>\varepsilon$ for some $x$. Then there is a $u \in E^{*}$ such that $\|u\| \leq 1$ and $|u(z(x))|>\varepsilon$. Now

$$
\begin{aligned}
u(z(x)) & =\int_{G}(u \circ f)(x-t) d \mu(t)-\sum_{i=1}^{n} \mu\left(E_{i}\right) \cdot(u \circ f)\left(x-t_{i}\right)= \\
& =\sum_{i=1}^{n} \int_{E_{i}}\left((u \circ f)(x-t)-(u \circ f)\left(x-t_{i}\right)\right) d \mu(t) .
\end{aligned}
$$

However, since $t-t_{i} \in V$ and $\left\|u(f(x-t))-u\left(f\left(x-t_{i}\right)\right)\right\|_{E}<\varepsilon /\|\mu\|$ whenever $t \in E_{i}$, we have $|u(z(x))| \leq \varepsilon$, which is impossible.

Now the function $\sum_{i=1}^{n} \mu\left(E_{i}\right) f\left(x-t_{i}\right)$ is in $L_{f}$. By (10) we find that $\mu * f$ can be uniformly approximated by functions from $L_{f}$, and thus $\mu * f \in V_{f}$.

If $f \in C(G, E)$ and $g \in C(G, \mathbb{C})$ then $f * g(x)$ is defined by $\int_{G} f(x-$ t) $g(t) d t$, where $d x$ is the Haar measure. Clearly, $f * g=\mu_{g} * f$, where $\mu_{g}(H)=$ $\int_{H} g d t$ for every Borel $H \subset G$. Thus $f * g \in V_{f}$.

It is clear that $C(G, E)$ is a Banach space with the norm $\|f\|=$ $\sup _{x \in G}\|f\|_{E}$. If $\Lambda \in C(G, E) *$ and $f \in C(G, E)$, then we put $\Lambda * f(x)=\Lambda\left(g_{x}\right)$, where $g_{x}$ is the function $t \mapsto f(x-t)(t \in G)$. Using the uniform continuity of $f$ it is easy to see that $\Lambda * f \in C(G, \mathbb{C})$. Our next aim is to show that if $\Lambda \in C(G, E)^{*}, f \in C(G, E)$ and $g \in C(G, \mathbb{C})$, then

$$
(\Lambda * f) * g=\Lambda *(f * g) .
$$

First we assume that the linear span $N(f)$ of $R(f)$ is of finite dimension. Let $e_{1}, \ldots, e_{n}$ be a basis of $N(f)$. Then there are functions $f_{i}: G \rightarrow \mathbb{C}$ such that $f=\sum_{i=1}^{n} f_{i} \cdot e_{i}$. There are linear functionals $u_{1}, \ldots, u_{k} \in E^{*}$ such that $u_{i}\left(e_{i}\right)=1$ and $u_{i}\left(e_{j}\right)=0$ for every $1 \leq i, j \leq k, i \neq j$. Thus $f_{i}=u_{i} \circ f$ for every $i=1, \ldots, k$. Since $f$ is continuous, we can see that so are $f_{1}, \ldots, f_{k}$.

Let $\Lambda_{i}(g)=\Lambda\left(g \cdot e_{i}\right)$ for every $g \in C(G, \mathbb{C})$ and $i=1, \ldots, n$. It is clear that $\Lambda_{i}$ is a bounded linear functional of $C(G, \mathbb{C})$. By the Riesz representation theorem, there are measures $\mu_{1}, \ldots, \mu_{n}$ such that $\Lambda_{i}(g)=\int_{G} g d \mu_{i}$ for every $g \in C(G, \mathbb{C})$ and $i=1, \ldots, n$. Then $\Lambda_{i} * g=\mu_{i} * g$ for every $g \in C(G, \mathbb{C})$ and 
$i=1, \ldots, n$, and thus

$$
\begin{aligned}
(\Lambda * f) * g & =\left(\Lambda * \sum_{i=1}^{n} f_{i} \cdot e_{i}\right) * g=\left(\sum_{i=1}^{n} \Lambda *\left(f_{i} \cdot e_{i}\right)\right) * g= \\
& =\left(\sum_{i=1}^{n} \Lambda_{i} * f_{i}\right) * g=\left(\sum_{i=1}^{n} \mu_{i} * f_{i}\right) * g= \\
& =\sum_{i=1}^{n}\left(\mu_{i} * f_{i}\right) * g=\sum_{i=1}^{n} \mu_{i} *\left(f_{i} * g\right)= \\
& =\sum_{i=1}^{n} \Lambda_{i} *\left(f_{i} * g\right)=\sum_{i=1}^{n} \Lambda *\left(\left(f_{i} \cdot e_{i}\right) * g\right)= \\
& =\Lambda *(f * g) .
\end{aligned}
$$

If $f \in C(G, E)$ is arbitrary, then a standard partition-of-unity argument ${ }^{3}$ shows that $f$ can be uniformly approximated by functions $f_{n}$ such that the linear span of $R\left(f_{n}\right)$ is of finite dimension for every $n$. Suppose $\left\|f-f_{n}\right\|_{E}<1 / n$ $(n=1,2, \ldots)$. Then $\left\|f * g-f_{n} * g\right\|_{E} \leq\|g\|_{1} / n$ on $G$,

$$
\begin{aligned}
\left|\Lambda *(f * g)-\Lambda *\left(f_{n} * g\right)\right| & \leq\|\Lambda\| \cdot\|g\|_{1} / n, \\
\left|\Lambda * f-\Lambda * f_{n}\right| & =\left|\Lambda *\left(f-f_{n}\right)\right| \leq\|\Lambda\| / n, \\
\left\|(\Lambda * f) * g-\left(\Lambda * f_{n}\right) * g\right\| & \leq\|\Lambda\| \cdot\|g\|_{1} / n .
\end{aligned}
$$

Since $\left(\Lambda * f_{n}\right) * g=\Lambda *\left(f_{n} * g\right)$, it follows that the norm of the difference of the two sides of (11) is at most $c / n$ for every $n$, where $c$ only depends on $\Lambda$ and $g$. This proves (11).

Let $\Gamma$ denote the dual of $G$. If $\gamma \in \Gamma$, then the invariance of the Haar measure gives

$$
(f * \gamma)(x)=\int_{G} \gamma(x-t) f(t) d t=e_{\gamma} \cdot \gamma(x),
$$

where $e_{\gamma}=\int_{G} \gamma(-t) f(t) d t$. Thus $e_{\gamma} \cdot \gamma \in V_{f}$ for every $\gamma \in \Gamma$. Each $\gamma \in \Gamma$ is an exponential. Thus linear combinations of the elements $e_{\gamma} \cdot \gamma$ are $E$-valued exponential polynomials by Theorem 4 . Therefore, it is enough to show that $f$ is in the closure of the set $N$ of linear combinations of the elements $e_{\gamma} \cdot \gamma$.

The closure $\operatorname{cl} N$ of $N$ is a closed subspace of $C(G, E)$, so if $f \notin \operatorname{cl} N$, then there is a $\Lambda \in C(G, E)^{*}$ such that $\Lambda(f) \neq 0$ and $\Lambda\left(e_{\gamma} \cdot \gamma\right)=0$ for every $\gamma \in \Gamma$. Let $M(g)=\Lambda(\hat{g})$, where $\hat{g}(x)=g(-x)$. Then $(M * g)(0)=\Lambda(g)$ for

\footnotetext{
${ }^{3}$ Let $\varepsilon>0$ be given. Let $V$ be a neighbourhood of 0 such that $\|f(x)-f(y)\|_{E}<\varepsilon$ whenever $x-y \in V$. Let $U$ be another neighbourhood of 0 such that $\operatorname{cl} U \subset V$, and let $\phi: G \rightarrow \mathbb{R}$ be a continuous function such that $\phi>0$ on $U, 0 \leq \phi \leq 1$ on $V$ and $\phi=0$ on $G \backslash V$. Let $x_{i} \in G(i=1, \ldots, n)$ be such that $G=\bigcup_{i=1}^{n}\left(U+x_{i}\right)$, and put $\Phi(x)=\sum_{i=1}^{n} \phi\left(x-x_{i}\right)$. Then $\Phi$ is a positive continuous function on $G$. Put $f_{\varepsilon}=\Phi^{-1} \cdot \sum_{i=1}^{n} \phi\left(x-x_{i}\right) \cdot f\left(x_{i}\right)$. Then $f_{\varepsilon} \in C(G, E)$, and the linear span of $R\left(f_{\varepsilon}\right)$ is of finite dimension. Now we have $f(x)-f_{\varepsilon}(x)=\Phi^{-1} \sum_{i=1}^{n} \phi\left(x-x_{i}\right) \cdot\left(f(x)-f\left(x_{i}\right)\right)$ for every $x$. If $x \notin V+x_{i}$, then $\phi\left(x-x_{i}\right)=0$. If $x \in V+x_{i}$, then $0 \leq \phi\left(x-x_{i}\right) \leq 1$ and $\left\|f(x)-f\left(x_{i}\right)\right\|_{E}<\varepsilon$, and thus $\left\|f(x)-f_{\varepsilon}(x)\right\|_{E}<\varepsilon$.
} 
every $g \in C(G, E)$. Therefore, we have $M * f \neq 0$ and $M *\left(e_{\gamma} \cdot \gamma\right)=0$ for every $\gamma \in \Gamma$. Then

$$
(M * f) * \gamma=M *(f * \gamma)=M *\left(e_{\gamma} \cdot \gamma\right)=0
$$

for every $\gamma \in \Gamma$. Since $M * f \in C(G, \mathbb{C})$ and the set of trigonometric polynomials is dense in $C(G, \mathbb{C})$, it follows that $M * f=0$, a contradiction.

\section{References}

[1] Aczél, J., Dhombres, J.: Functional Equations in Several Variables. Encyclopedia of Mathematics and Its Applications, vol. 31. Cambridge University Press, Cambridge (1989)

[2] Bochner, S., von Neumann, J.: Almost periodic functions in groups. II. Trans. Am. Math. Soc. 37, 21-50 (1935)

[3] Devaraj, P., Rana, Inder K.: Vector valued mean-periodic functions on groups. J. Aust. Math. Soc. 72(3), 363-388 (2002)

[4] Diestel, J.: Sequences and Series in Banach Spaces Graduate Texts in Mathematics, vol. 92. Springer, New York (1984)

[5] Djoković, D.Ž.: A representation theorem for $\left(X_{1}-1\right)\left(X_{2}-1\right) \ldots\left(X_{n}-1\right)$ and its applications. Ann. Polon. Math. 22, 189-198 (1969)

[6] Laczkovich, M.: Polynomial mappings on Abelian groups. Aequationes Math. 68, 177-199 (2004)

[7] Laczkovich, M.: Local spectral synthesis on Abelian groups. Acta Math. Hungar. 142, 313-329 (2014)

[8] Laczkovich, M.: A characterization of generalized exponential polynomials in terms of decomposable functions. Acta Math. Hungar. 158(2), 338-351 (2019)

[9] Laczkovich, M.: The Levi-Civita equation in function classes. Aequationes Math. 94(4), 689-701 (2020)

[10] Laczkovich, M., Székelyhidi, G.: Harmonic analysis on discrete Abelian groups. Proc. A.M.S. 133(6), 1581-1586 (2005)

[11] Laczkovich, M., Székelyhidi, L.: Spectral synthesis on discrete Abelian groups. Math. Proc. Camb. Phil. Soc. 143, 103-120 (2007)

[12] McKiernan, M.A.: Equations of the form $H(x \circ y)=\sum_{i} f_{i}(x) g_{i}(y),$. Aequationes Math. 16, 51-58 (1977)

[13] Rudin, W.: Functional Analysis. McGraw-Hill, New York (1973)

[14] Székelyhidi, L.: Note on exponential polynomials. Pac. J. Math. 103, 583-587 (1982)

[15] Székelyhidi, L.: Convolution Type Functional Equations on Topological Abelian Groups. World Scientific, Singapore (1991)

[16] Székelyhidi, L.: The failure of spectral synthesis on some types of discrete Abelian groups. J. Math. Anal. Appl. 291, 757-763 (2004) 


\section{Laczkovich}

ELTE Eotvos Lorand University

Budapest

Hungary

e-mail: miklos.laczkovich@gmail.com

Received: April 20, 2020.

Accepted: September 11, 2020.

Publisher's Note Springer Nature remains neutral with regard to jurisdictional claims in published maps and institutional affiliations. 